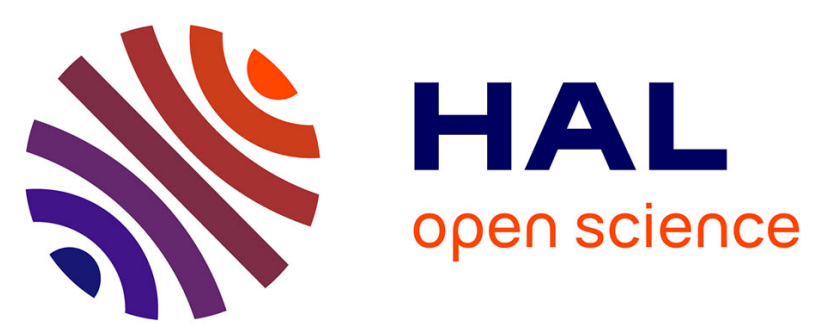

\title{
CMA-Based Blind Equalization and Phase Recovery in OFDM/OQAM Systems
}

Vincent Savaux, Faouzi Bader, Jacques Palicot

\section{To cite this version:}

Vincent Savaux, Faouzi Bader, Jacques Palicot. CMA-Based Blind Equalization and Phase Recovery in OFDM/OQAM Systems. 2016 23rd International Conference on Telecommunications (ICT), May 2016, Thessaloniki, Greece. 10.1109/ict.2016.7500466 . hal-01311054

\section{HAL Id: hal-01311054 \\ https://hal-centralesupelec.archives-ouvertes.fr/hal-01311054}

Submitted on 1 Jun 2016

HAL is a multi-disciplinary open access archive for the deposit and dissemination of scientific research documents, whether they are published or not. The documents may come from teaching and research institutions in France or abroad, or from public or private research centers.
L'archive ouverte pluridisciplinaire HAL, est destinée au dépôt et à la diffusion de documents scientifiques de niveau recherche, publiés ou non, émanant des établissements d'enseignement et de recherche français ou étrangers, des laboratoires publics ou privés. 


\title{
CMA-Based Blind Equalization and Phase Recovery in OFDM/OQAM Systems
}

\author{
*Vincent Savaux, **Faouzi Bader, and **Jacques Palicot \\ **SCEE/IETR - CentraleSupélec, Rennes. \\ Avenue de la Boulaie, 35576 Cesson - Sévigné Cedex, France. \\ Emails: faouzi.bader@supelec.fr, and jacques.palicot@centralesupelec.fr
}

\begin{abstract}
This paper deals with a constant modulus algorithm(CMA) based blind equalization method for orthogonal frequency division multiplexing/offset quadrature amplitude modulation (OFDM/OQAM) scheme. The authors propose to combine the CMA with a low-pass filter in order to increase the convergence speed of the equalizer, and to avoid the detection of the received symbols with phase mismatch. Simulations results show that the convergence speed is largely increased, and the proposed method achieves bit error rate (BER) performance very close to that of the perfect channel estimation and equalization.
\end{abstract}

Index Terms-Blind equalization, OFDM/OQAM, CMA, Impulse Noise Cancellation.

\section{INTRODUCTION}

The blind equalization is an interesting solution for the channel inversion (or deconvolution) since it guarantees the signal a maximum spectral efficiency. Indeed, these techniques do not use pilots, the blind equalizer only takes advantage of a few signal features to perform the channel inversion. In [1], B. Farhang-Boroujeny has adapted the blind equalization using the constant modulus algorithm (CMA) proposed by D. $\mathrm{N}$. Godard in [2] to the cosine modulated filter bank (CMFB) modulation. In [3] the authors proposed to use the second order moment of the received signal, while the CMA is used in [1].

In filter bank multicarrier (FBMC), and in particular in orthgonal frequency division multiplexing/offset quadrature amplitude modulation (OFDM/OQAM), only few papers deals with blind equalization. Due the convergence delay of the blind methods and the uncertainty on the phase of the recovered symbols, the blind channel equalization remains a pending challenge not much studied by the scientific community, in particular in FBMC.

In this paper we propose a constant modulus algorithmbased (CMA) blind equalizer for OFDM/OQAM systems. As in [1], we consider a one-tap per carrier equalization, since it is particularly adapted to the multicarrier systems. As mentioned in [4], this type of technique may lead to local solutions and implies that the symbols are detected with a phase shift of 0 or $\pi$. The proposed method consists of filtering the equalizer response in order to smooth the channel frequency response. The principle of the proposed technique is simple, since it is similar to a low-pass filter as used in channel estimation [5]. The addition of a differential phase coding allows to suppress any uncertainty on the phase of the recovered symbols.

*CentraleSupélec is the former institution of Vincent Savaux, actually with $\mathrm{b}<>$ com research institute at: vincent.savaux@b-com.com.
Furthermore, the proposed techniques largely increases the convergence speed of the CMA. The idea behind CMA applied in OFDM/OQAM is to iteratively update the algorithm by comparing the real part of the output of the equalizer with a given real constant that depends on the constellation size. However, it is worth noting that CMA is equivalent to Sato's algorithm [6], and the constant norm algorithm (CNA) [7], due to the use of real transmitted OQAM symbols. Therefore, this limits the choice of the cost function to those that are adapted to real constellation, whereas numerous cost functions adapted to different complex constellations have been proposed in the scientific literature [7]-[9].

The rest of the paper is organized as follows: Section II presents the system model and the CMA, and we propose our equalizer with IN cancellation in Section III. Simulations shows the performance of the method in terms of mean square error (MSE) and BER in Section IV, and we draw conclusions in Section V.

\section{System Model}

We consider the transmission of OFDM/OQAM symbols over a frequency selective channel, with perfect time and frequency synchronization. At the frequency-time position $(m, n)$, the output of the analysis filter bank (AFB) is written as

$$
y_{m, n}=H_{m, n} x_{m, n}+\underbrace{j \sum_{(p, q) \in \Omega} H_{p, q} x_{p, q}<g>_{p, q}^{m, n}}_{I_{m, n}}+w_{m, n},
$$

where $x_{m, n}$ is the real symbol transmitted at frequencytime position $(m, n), H_{m, n}$ is the complex channel frequency response (CFR). A detailed description of the synthesis filter bank (SFB) and the AFB is available in [10]. $w_{m, n}$ and $I_{m, n}$ are the complex additive white Gaussian noise (AWGN) and the interference terms whose variances are $\sigma^{2}$ and $\sigma_{I}^{2}$ respectively. The term $j<g>_{p, q}^{m, n}$ is called the intrinsic interference due to the prototype filter $g_{m, n}(t)$ that is given by

$$
g_{m, n}[i]=g\left(i-n \frac{M}{2}\right) e^{\frac{2 j \pi m}{M}\left(i-\frac{L_{f}-1}{2}\right)} e^{j \phi_{m, n}},
$$

where the phase term is $\phi_{m, n}=(\pi / 2)(m+n)+m n \pi$ as defined in [10]. It should be noted that the real symbols $x_{m, n}$ 
are transmitted with a period $\frac{M}{2}$ twice shorter than that of OFDM signal where symbols are complex. The used filter has a length $L_{f}=K M$, where $K$ is the overlapping factor and $M$ is the total number of carriers. Thus, even in absence of the multipath channel, there will be some intercarrier and intersymbol purely imaginary interferences. In fact, $g_{m, n}$ is orthogonal in the real field, which allows to perfectly recover the real OQAM symbols. However, it can be seen in (1) that the CFR $H_{p, q}$ induces some distortions from the timefrequency positions $(p, q)$ that belongs to the set $\Omega^{1}$ and have to be estimated and corrected.

In this paper, the signal is blindly equalized, by using the simple one-tap per-carrier CMA equalizer. To summarize, the aim of the CMA is to tends toward the optimal equalizer coefficient $F_{m, n}^{o p t}$ thanks to the update algorithm, which can be written as

$$
F_{m, n+1}=F_{m, n}-\mu y_{m, n}^{*} \operatorname{sign}\left(a_{m, n}\right)\left(\left|a_{m, n}\right|-\gamma\right) .
$$

where $F_{m, n}$ is the equalizer coefficient, and $a_{m, n}=$ $\mathfrak{R e}\left(F_{m, n} y_{m, n}\right), \mu$ is the step-size parameter, and $\gamma$ is a realvalued constant which is defined as $\gamma=\frac{E\left\{|x|^{4}\right\}}{E\left\{|x|^{2}\right\}}$ (see [2] for more details). The CMA has a low complexity, but it suffers from two drawbacks: i) it requires a long convergence time, and ii) it is phase blind, i.e. the real equalized symbols $a_{m, n}$ can be detected with a phase equal to 0 or $\pi$. In the following, we propose a simple method that reduces the delay of convergence of CMA, and avoids the phase incertainty.

\section{CMA Blind Equalization With Impulse Noise CANCELLATION}

It has been shown in [1], [4] that the CMA applied to the real OQAM symbols converges toward the optimal coefficient value $\left|F_{m, n}^{o p t}\right|$ with a phase shift equal to 0 or $\pi$. The solution that consists of using a differential phase coding as in [1] do not ensure a low bit error rate (BER) value when a large number of received symbols are detected with an opposite phase. In this section, we propose to consider the issue of convergence toward $F_{m, n}^{o p t}$ or $-F_{m, n}^{o p t}$ from an original point of view, which is described just below.

\section{A. Equalization with Impulsive Noise Cancellation}

Fig. 1 shows the real part of a frequency response of the invert of the equalizer coefficients $1 / F_{m, n}^{o p t}$ (using CMA) compared with the exact channel frequency response $H_{m, n}$, knowing that $\left|F_{m, n}^{o p t} H_{m, n}\right|=1$. It can be seen that the frequency positions $m=11,29$, and 92 correspond to the opposite minima $-F_{m, n}^{o p t}$.

The optimal solutions with opposite phase depicted in Fig. 1 can be seen as an impulsive noise (IN) in the channel frequency response, which can be filtered in a simple way as described in [11]. The IN cancellation can be seen as a low-pass filtering, but performed in the time domain. This technique is also usually used in channel estimation techniques in order to filter the noise or to perform an interpolation [5]. In

\footnotetext{
${ }^{1} \Omega$ is the set of positions $p, q$ that surround the position $m, n$
}

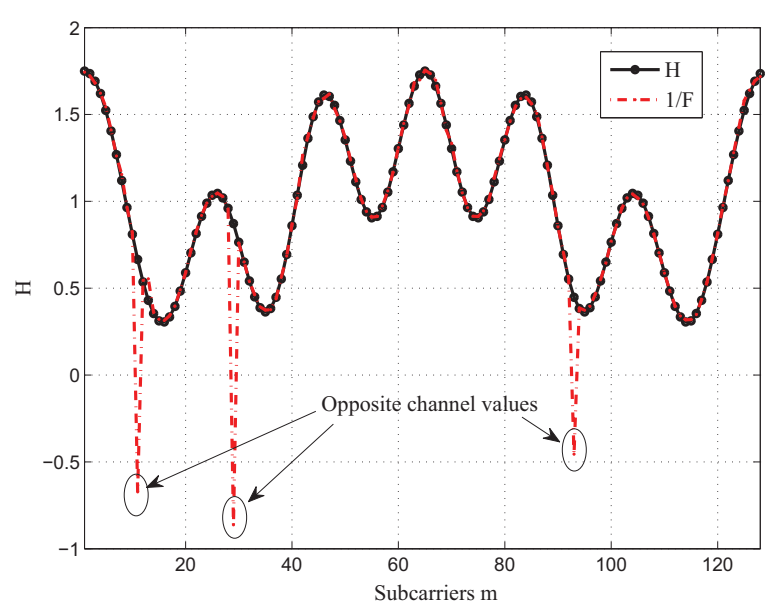

Fig. 1. Real part of $H$ and $1 / F$ showing opposite equalizer coefficient values.

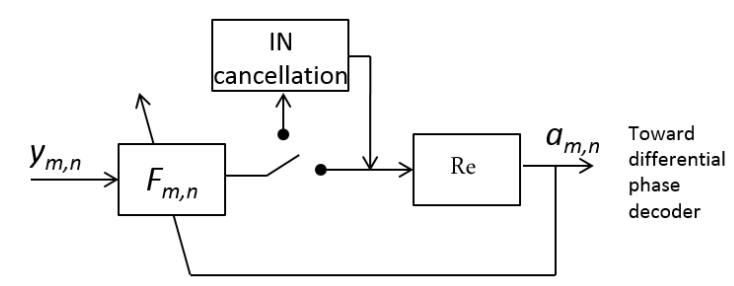

Fig. 2. Proposed equalizer structure with impulse noise cancellation.

the considered blind equalization context, the IN cancellation should be seen as a smoothing stage applied to the equalizer coefficients $F_{m, n}$, and allows full blind equalization as it does not require any pilot for the phase estimation. The proposed equalizer regularly switch to the IN cancellation block as depicted in Fig. 2.

The steps into the "IN cancellation" block in Fig. 2 are described as follows:

1) $\forall m \in \llbracket 0, M-1 \rrbracket$, the channel frequency response (CFR) samples are obtained by $\hat{H}_{m, n}=1 / F_{m, n}$, and stored in the vector $\hat{\mathbf{H}}_{n}$ of size $M \times 1$.

2) The estimated channel impulse response (CIR) is achieved by applying an IFFT: $\hat{\mathbf{h}}_{n}=\operatorname{IFFT}\left(\hat{\mathbf{H}}_{n}\right)$.

3) The CIR is multiplied by a rectangular window $\Pi$ defined by

$$
\Pi_{m}=\left\{\begin{array}{ll}
1, & \text { if } m \leq L_{\Pi} \\
0, & \text { else }
\end{array},\right.
$$

such as $\overline{\mathbf{h}}_{n}=\hat{\mathbf{h}}_{n} \Pi$, where the length of $\Pi$ denoted by $L_{\Pi}$ must be at least equal to channel length $L$.

4) The filtered CFR is obtained by the FFT as $\overline{\mathbf{H}}_{n}=$ $\operatorname{FFT}\left(\overline{\mathbf{h}}_{n}\right)$ and then the equalizer coefficients by $F_{m, n}=$ $1 / \bar{H}_{m, n}$.

Note that in practice, the $L$ value is unknown, so $L_{\Pi}$ is chosen in order to overestimate the length of the channel. The advantages of the IN cancellation process are multiple: in addition to suppress the opposite solutions that are seen as an impulsive noise, it also avoids the possible convergence 
of the coefficients toward local minima ${ }^{2}$. Therefore, it offers the possibility of initialization strategies different from the one proposed in [1]. Moreover, as observed in [5] for the channel estimation, the IN cancellation mitigates the noise and the interferences level. In an OFDM/OQAM context in which the interference term may be not negligible, this smoothing stage may appear to be very convenient.

Also, it is worth highlighting that the proposed IN cancellation process using scheme in Fig. 2 may lead to two solutions: i) all the $M$ coefficients $F_{m, n}$ converge toward their optimal solutions without phase shift $F_{m, n}^{o p t}$, ii) all the coefficients $F_{m, n}$ are equal to the opposite expected solutions $-F_{m, n}^{o p t}$.

That is the main difference with the CMA in (3) where each coefficient $F_{m, n}$ may converge to the optimal value or its opposite. In our solution, if all the coefficients $F_{m, n}$ converge to their opposite optimal solution, the differential phase decoding stage leads to a single error ${ }^{3}$ which could be easily suppressed by means of a channel code. If no IN cancellation is performed as in [1], a large number of phases may be erroneous because they are detected with a difference equal to $\pi$. In the latter case, the differential phase encoding becomes inefficient and therefore the channel coding as well. In the hereafter section, we will focus on the way of applying the proposed IN cancellation stage in Fig. 2.

\section{B. How to apply the IN Cancellation?}

There are three possible ways to perform the IN cancellation stage, which are hereafter explained:

- The IN cancellation is used only one time during the convergence process. It could be a convenient solution if only few $F_{m, n}$ coefficients are detected with an opposite phase (see the example in Fig. 1). However, if a large number of phases are detected with a shift equal to $\pi$, a single IN cancellation stage is not sufficient to suppress the appearance of the impulse noise and this solution is similar to the CMA approach. Furthermore, this option raises the issue of the choice of the appropriate starting point to proceed the smoothing stage.

- The IN cancellation is employed at each iteration. Here, the update algorithm (3) does not have any sense since it is fed by a coefficient $F_{m, n}$ that is not the one obtained at the previous iteration. In other words, this solution is not anymore a blind equalization, and should lead to unsuitable coefficients $F_{m, n}$.

- The operation is performed with a reasonable regularity regarding to the convergence speed of the update algorithm (3). Thus, the process either performs the IN cancellation as long as optimal solutions with opposite phases remain, or performs a noise cancellation due to the nature of the filter. This solution seems to be the way to carry out the proposed CMA with IN cancellation in Fig. 2.

\footnotetext{
${ }^{2}$ In fact, local minima can also be seen as peaks in the estimated CFR

${ }^{3}$ If all the phases are detected with an error equal to $\pi$, the difference of phase between to consecutive symbols is the same as if the phase error is 0 , excepted for the first symbol.
}

In order to formalize these three solutions, we define $N_{i}$ the time delay between two consecutive smoothing operations. Thus, the first solution corresponds to $N_{i}=+\infty$, the second one to $N_{i}=1$ and the third one to a value of $N_{i}$ conveniently chosen. It is worth noticing that simulations revealed that the first two solutions lead to unsatisfactory results. As a consequence, we only focus on the third solution in the next section.

\section{Simulations Results}

In this section, we present simulations results which show the performance of the proposed blind equalizer. Since we are comparing obtained results with the CMA in (3), we will use the same parameters as described in [1] where the CMA is used in FBMC. We consider a constant multipath channel whose CIR is $\mathbf{h}=[1,-0.2,0.3,0.2,0.1,0.2,0.35,-0.2]$. The real part of the corresponding CFR is displayed in Fig. 1. The results are presented for a 4-QAM constellation, i.e. for binary OQAM symbols. As a consequence, note that $\gamma=1$. Each OFDM/OQAM symbol is composed of $M=$ 128 carriers. Two initialisation values are tested: a constant initialization $F_{m, 0}=0.01$ for any $m=0,1, . ., M-1$ such as proposed in [1], and the sub-optimal initialization strategy $F_{m, 0}^{s-o p t}=\sqrt{\frac{E\left\{x_{m, 0}\right\}^{2}}{\left|y_{m, 0}\right|^{2}}}$ such as proposed in [12]. In both cases, the step-size parameters is set at $\mu=0.015$. In the following, the performance of the data-aided interference approximation method (IAM) is also plotted for reference. A detailed description of the IAM is provided in [10]. The performance of the different blind equalizers is assessed by using the mean square error MSE defined as

$$
\mathrm{MSE}=E\left\{\left(\left|a_{m, n}\right|-\left|x_{m, n}\right|\right)^{2}\right\} .
$$

In Fig. 3, the CMA is compared with the proposed CMA using IN cancellation (denoted by INC in the figures) for $N_{i}=$ 1 and $N_{i}=+\infty$, where it is performed at the 200th and at the 2000th iterations. The results are obtained for $\mathrm{SNR}=30$ $\mathrm{dB}$, and $F_{m, 0}=0.01$. Moreover, achieved MSE values with IAM pilot aided pattern is plotted as a reference.

It can be observed that CMA achieves better performance than CMA with IN cancellation when it is performed with $N_{i}=1$ and has a similar MSE than CMA using IN cancellation where $N_{i}=+\infty$. Having $N_{i}=1$, the MSE has a "chaotic" behavior, but has a lower bound around $-14 \mathrm{~dB}$, whereas the CMA achieves a MSE lower than $-17 \mathrm{~dB}$. These observations confirm that the gradient update in (3) does not make sense in the case $N_{i}=1$. For $N_{i}=+\infty$, one can observe that once the smoothing stage has been performed (at the 200th or 2000th iteration), the trajectories of CMA using IN cancellation are the same as those with CMA. The peaks that appear at iterations 200 and 2000 can be interpreted as a reset of the equalizer coefficient due to the IN cancellation stage, and this indicates that the option $N_{i}=1$ is not suitable when too much coefficients $F_{m, n}$ converge to their opposite solutions. More generally, results presented in Fig. 3 show that the IN cancellation with $N_{i}=1$ and $N_{i}=+\infty$ does not improve CMA as proposed in [1]. These results are consistent with the a priori remarks we made in Section III-B. Note that 


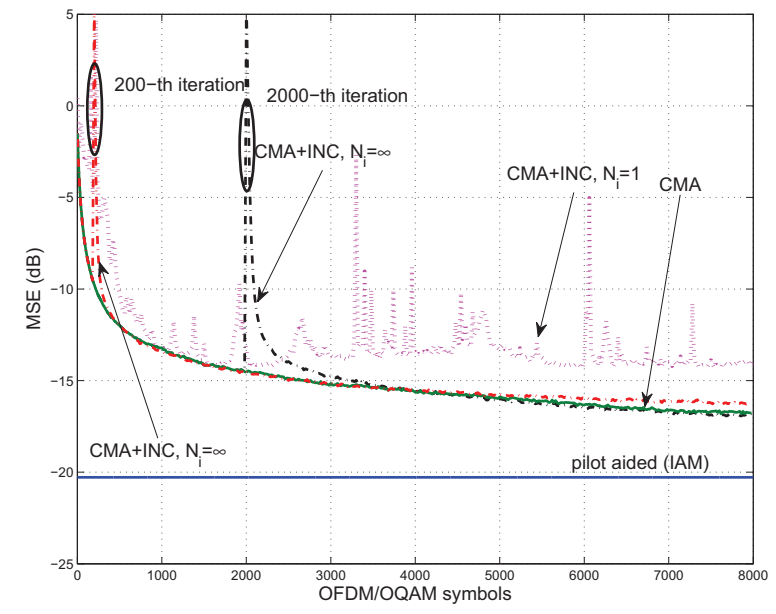

Fig. 3. MSE of CMA and CMA with impulse noise cancellation (INC) versus OFDM/OQAM symbols for $N_{i}=1$ and $N_{i}=+\infty, \mathrm{SNR}=30 \mathrm{~dB}$.

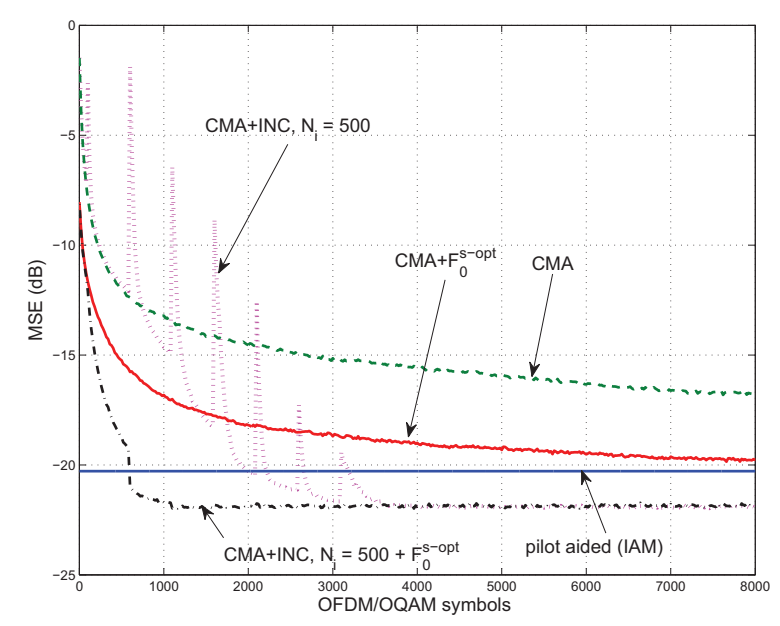

Fig. 4. MSE of the CMA using IN cancellation versus iterations compared with $\mathrm{CMA}, N_{i}=500, \mathrm{SNR}=30 \mathrm{~dB}$.

none of the presented methods is able to reach the performance of the pilot-aided estimation using IAM. This is mainly due to the facts that: i) IAM is an accurate channel estimation method, and ii) CMA is known to be a simple method, but achieves weak performance.

In Fig. 4 we compare the MSE performance of the proposed CMA in Fig. 2 for a regular IN mitigation stage performed every $N_{i}=500$ iterations. Furthermore, the initialization $F_{m, 0}^{s-o p t}$ is compared with the constant $F_{m, 0}=0.01$ value.

It can be observed that CMA with sub-optimal initialization achieves a gain of 2000 iteration at $\mathrm{MSE}=-15 \mathrm{~dB}$ compared with the "classical" CMA such as proposed in [1]. Fig. 4 also highlights that the CMA using IN cancellation with $N_{i}=500$ reaches its lower bound equal to $\mathrm{MSE}=-22 \mathrm{~dB}$ at the 3500th iteration, and when $F_{m, n}^{s-o p t}$ is used, this MSE floor is reached at the 1000-th iteration. These results show the capability of the proposed CMA-based equalizer with convenient

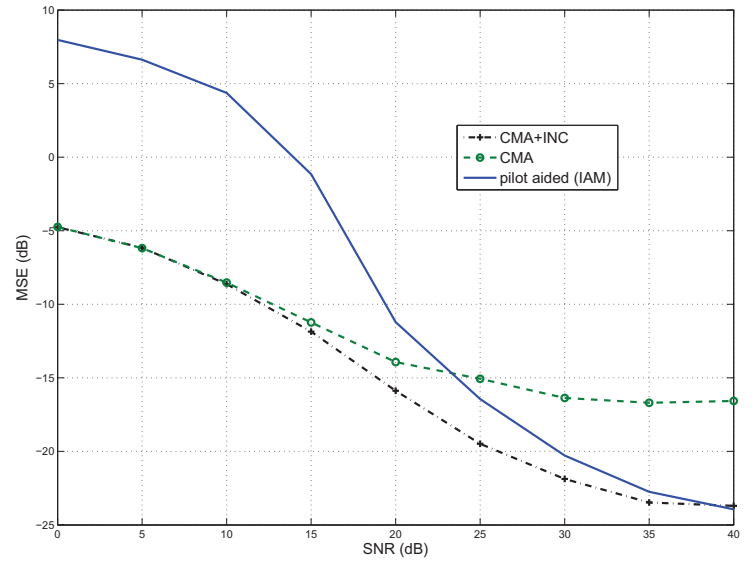

Fig. 5. MSE performance versus SNR. Comparison between CMA, CMA using IN cancellation (ICN) with sub-optimal initialization value, and pilotaided method (IAM).

$N_{i}$ value to have a high convergence speed compared with CMA. Furthermore, it must be noticed that this advantage is accompanied with a good performance, as a MSE gain of 6 $\mathrm{dB}$ is achieved by the proposed method compared to the CMA at the 4000-th iteration. Also, it can be seen that CMA with IN cancellation achieves a gain of $2 \mathrm{~dB}$ of MSE compared with the pilot-aided method.

For proposed CMA approach with IN cancellation, one can notice that peaks appear at the iterations corresponding to the application of IN cancellation stages. This is due to the fact that the IN cancellations gradually suppress the peaks in the equalizer coefficients. Although the proposed equalizer in Fig. 2 with the initialization in $F_{m, 0}^{s-o p t}$ has a remarkable convergence speed, it can be observed that the initialization $F_{m, 0}^{s-o p t}$ alone with the CMA allows to get a satisfactory fast convergence as well, such as shown in [12]. However, the MSE defined in (5) does not take into account the phase shift of 0 or $\pi$. In fact it does not affect the absolute values of the expected equalizer coefficients $\left|F_{m, o p t}\right|$ but plays a key role in the BER performance, as it is shown afterward.

Fig. 5 depicts the MSE-floor values of CMA, and CMA using IN cancellation with sub-optimal initialization value, and IAM, when the steady-state is reached, versus SNRs. Once again, it can be concluded from Fig. 5 that the blind equalizers achieve better performance than the data aided method (at least for the same channel as in [1]) for SNR values $<24 \mathrm{~dB}$ using CMA and $<40 \mathrm{~dB}$ using CMA with IN cancellation $\left(N_{i}=\right.$ 500). Furthermore, the latter has lower MSE values than the "usual" CMA for SNR $>10 \mathrm{~dB}$. This result reflects the fact that the IN cancellation stage is also able to smooth the noise of the channel frequency response (step 3) in the algorithm presented in Section III.

Fig. 6 shows the BER performance of the proposed blind equalizer versus the SNR, and also that of CMA approach using both the constant initialization and $F_{m, 0}^{s-o p t}$. The BER is obtained by using a differential phase coding and a convolutional coding with code rate $1 / 2$ and a Viterbi decoder. 


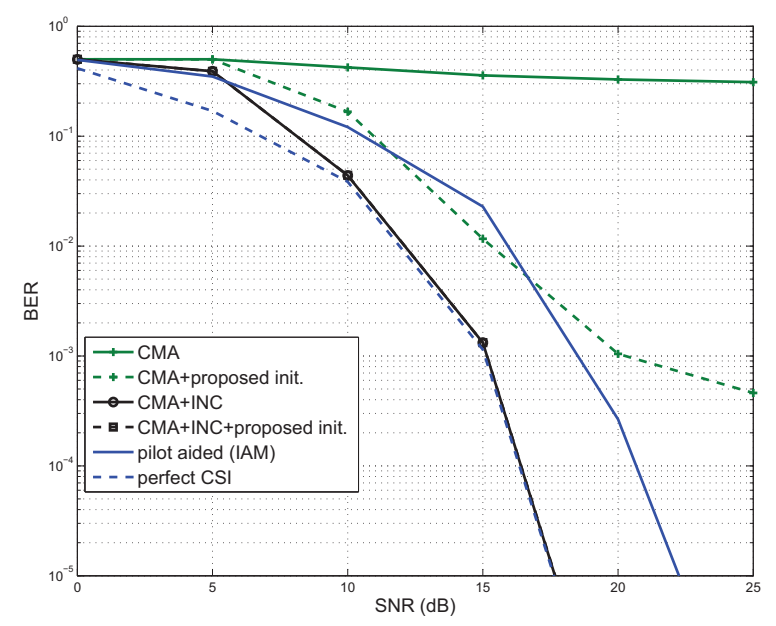

Fig. 6. BER versus SNR of the proposed equalizer compared to [1].

The BER is calculated at the 4000th iteration, by averaging the results on $10^{7}$ random transmitted bits. The data aided estimation and equalization using IAM is also depicted, and the equalizer using perfect CSI is plotted as a reference.

It can be clearly observed that the proposed technique outperforms the blind equalizer using CMA proposed in [1]. This reflects the fact that a large number of coefficients $F_{m, n}$ converge toward their opposite optimal values. Consequently, when CMA is used alone, a large number of symbols are detected with a phase shift equal to $\pi$. In that case, the differential phase decoder and the Viterbi decoder are unable to recover the expected correct binary symbols. With the proposed blind equalizer, this problem is solved since all the symbols are detected with the correct phase thanks to both the differential and Viterbi decoders. Moreover, it should be noticed that a SNR gain of $3 \mathrm{~dB}$ is achieved by the CMA with IN cancellation compared with the pilot aided estimation and equalization, which is consistent with the MSE result. Furthermore, the CMA with IN cancellation losses less than $0.2 \mathrm{~dB}$ compared with the equalizer with perfect CSI. This is an important result since it shows that blind equalization (once the steady-state is reached) is able in our context to outperform data-aided techniques, in terms of both BER performance and spectral efficiency.

Fig. 6 also reveals that the use of the sub-optimal initialization has almost no influence on the BER performance. However, it has been shown that largely improves the convergence speed of the equalizer. From the performance analyzed in both Figs. 4 and 6, we conclude that the combination of the proposed method with the initialization $F_{m, 0}^{s-o p t}$ leads to i) a fast equalizer, ii) a low MSE floor value, and iii) a high BER performance, compared with CMA alone, and with IAM. Furthermore, the proposed with IN cancellation allows to achieve a full blind equalizer and phase recovery, since no pilot is used.

\section{CONCLUSION}

In this paper, we presented a simple blind equalization technique for OFDM/OQAM systems. The principle is to combine the CMA and a low-pass filtering in order to increase the convergence speed of the blind equalizer, and to avoid the convergence of the coefficients toward wrong phase values. Simulations revealed that the proposed method achieves performance close to the perfect channel estimation and equalization. Further work will deal with the extension of proposed method to larger constellation sizes.

\section{ACKNOWLEDGMENT}

This work is supported by the project PROFIL (Evolution of the wideband PROfessional Mobile Radio based on the FILter Bank MultiCarrier modulation) funded by the French national research agency (ANR) with grant agreement code: ANR-13INFR-0007-03.

\section{REFERENCES}

[1] B. Farhang-Boroujeny, "Multicarrier Modulation With Blind Detection Capability Using Cosine Modulated Filter Banks," IEEE Transactions on Communications, vol. 51, no. 12, pp. 2057 - 2070, December 2003.

[2] D. N. Godard, "Self-Recovering Equalization and Carrier Tracking in Two-Dimensional Data Communication Systems," IEEE Transactions on Communications, vol. com-28, no. 11, pp. 1867 - 1875, November 1980.

[3] H. Bölcskei, P. Duhamel, and R. Hleiss, "A Subspace-Based Approach to Blind Channel Identification in Pulse Shaping OFDM/OQAM Systems," IEEE Transactions on Signal Processing, vol. 49, no. 7, pp. $1594-1598$, July 2001

[4] L. Lin and B. Farhang-Boroujeny, "Convergence analysis of blind equalizer in a cosine modulated filter bank-based multicarrier communication system," in proc. of SPAWC'03, Roma, Italy, June 2003, pp. $368-372$.

[5] J. Schoukens, R. Pintelon, and H. V. Hamme, "The Interpolated Fast Fourier Transform: A Comparative Study," IEEE Transactions on Instrumentation and Measurement, vol. 41, no. 2, pp. 226 - 232, April 1992.

[6] Y. Sato, "A Method of Self-Recovering Equalization for Multilevel Amplitude-Modulation Systems," IEEE Transactions on Communications, vol. 23, no. 6, pp. 679 - 682, June 1975.

[7] A. Goupil and J. Palicot, "New Algorithms for Blind Equalization: The Constant Norm Algorithm Family," IEEE Transactions on Signal Processing, vol. 55, no. 4, pp. 1436 - 1444, April 2007.

[8] K. N. Oh and Y. O. Chin, "Modified constant modulus algorithm: blind equalization and carrier phase recovery algorithm,' in proc. of ICC'95, vol. 1, Seattle, WA, June 1995, pp. $498-502$.

[9] S. Barbarossa, "Blind Equalization Using Cost Function Matched to the Signal Constellation," in proc. of ACSSC'97, vol. 1, Pacific Grove, CA, USA, November 1997, pp. 550 - 554.

[10] E. Kofidis, D. Katselis, A.Rontogiannis, and S. Theodoridis, "Preamblebased channel estimation in OFDM/OQAM systems: A review," Signal Processing, Elsevier, vol. 93, pp. 2038 - 2054, January 2013.

[11] S. V. Vaseghi, Advanced Digital Signal Processing and Noise Reduction. John Wiley \& Sons, 2000, ch. 12, pp. $355-377$.

[12] V. Savaux and F. Bader, "Sub-Optimal Initialization for Blind Equalization with Fast Convergence in OFDM/OQAM Modulation," in proc. of EuCNC'15, Paris, France, June - July 2015. 